\title{
Health Monitoring using E-Health Sensors and the Cloud
}

\author{
Mangalagouramma A. Shivasimpiger and Dr.S.M. Joshi
}

\begin{abstract}
The combination of computer science and electronics has resulted into one of the most notable technological revolutions in the form of Internet of Things(IoT).The implementation of IoT in healthcare has lead to inexpensive, non-invasive and ambulatory health monitoring. This paper introduces a technique that uses IoT and the cloud computing to implement better health monitoring by preventing delays in the arrival of patient's health information to the hospitals. This can be helpful in case of accidents and emergencies because there is no need of entering data manually and the data is made available automatically from e-health sensors to the medical staff by using cloud application software (Dropbox).
\end{abstract}

Keywords--- Ambulatory Health Monitoring, Cloud computing, E-Health Sensors, Internet of Things.

\section{INTRODUCTION}

$\mathrm{I}_{\mathrm{c}}^{\mathrm{N}}$ NTERNET of Things : The computer and the internet are completely dependent on the human operators for information. The problem of such a system is that people have limited time, accuracy and attention; meaning that the people are not good at capturing data. One solution to this problem may be to make devices smart and with the emerge of Internet of Things; the devices are independently able to connect and communicate with each other without the human intervention. These smart devices gather the information on their own.

Internet of Things technology can be used in transforming of information from the world of physical devices into the world of digital 0 's and 1's. The medical devices used gather the information and share the same information directly with each other and with the cloud. The cloud makes it possible to collect the data, record the data and analyze the data of patient in order to improve the patient's health. IoT has 3 views:

1) Things Oriented View

2) Internet Oriented View

3) Semantic Oriented View

a) Things Oriented View: It provides the view that all the real physical objects can get attached to one of the sensors to access the real time information from those objects. The same can be implemented by the sensors board network of embedded physical devices using different wireless technologies. This view represents integration of "things".

Mangalagouramma A. Shivasimpiger, M.Tech Student, Department of Computer Science and Engineering, SDMCET, Dharwad, VTU, Karnataka,India.Email:mangalagouramma@gmail.com

Dr.S.M. Joshi, Associate Professor, HOD, Dept. of CSE, SDMCET, Dharwad.

DOI:10.9756/BIJSESC.8264 b) Internet Oriented View: It provides the illusion that all the physical devices can get connected through internet and can be called as smart devices. This can be achieved by using unique Internet Protocol address for each connected object in the internet. This view represents the "integration of data from all the smart objects" and this data can be continuously monitored.

c) Semantic Oriented View: This view provides the perspective (illusion) that the data collected from different sensors need to be analyzed for necessary interpretation. This can be implemented with semantic techniques and middleware that separates unwanted data from meaningful data.

Cloud Computing: It is a term that ensures about delivery of any and all the information technology from a computing infrastructure i.e. resources to a computing power, its applications and business processes to end users as a service whenever and wherever they require it[1].

In this proposed work one of the categories of cloud offerings known as SaaS(i.e Storage as a Service) is used to store health information. This is implemented using Dropbox Application software that provides space for storing patient's information for further analysis.

The cloud delivery model used in this proposed work is the public cloud wherein the health information is being stored. In the public cloud the data is accessible to all users[1]. In this proposed work the Dropbox application software makes use of Gmail user Id and password to provide security and access control to the stored data.

\section{LITERATURE SURVEY}

The elder population is growing and the younger population is aging and the $85 \%$ of the population is living with chronic diseases such as cancer, heart related diseases. The use of IoT in the health monitoring has made very much impact on a patient's health condition.

According to a technical paper "transforming health care through IoT “, published in "Work Management Practioner's Conference 2014" chapter there will be approximately 26 billion physical devices on the "Internet of Things" by 2020 .

This paper provides evidence that the physiological information received from physical wireless devices could be a great contributor for managing and preventing deadly diseases, at the same time it also helps in analyzing the patient's condition after hospitalization.

Case Study 1: The "Vsee telemedicine team setup the eye clinic at the Syrian Refugee Camp in Duhok. The team was provided with telemedicine field kit consisting of stethoscope, 
heart rate, blood pressure, ultrasounds etc. connected with the doctor who is in a remote location. These devices transmitted data to the doctor in a real time along with video conferencing technique. The team treated more number of refugee patients and performed surgeries within very few hours.

This method of treatment resulted in

a) Controlling crowd at the local hospitals and feed up more appointments for urgent or serious cases.

b) The remotely handled health care's quality was as equal to personal visit to the hospitals.

c) Patients from rural areas felt this as a more convenient treatment, avoiding frequent and long distant journeys to reach to a doctor's place.

But this real time work faced many challenges of limited memory, limited computing power and limited energy.

Case Study 2: According to the paper "Health care applications of the IoT : a review ", IoT is the technique that helps in achieving excellent health care and monitoring of patient's condition at affordable costs.

This paper also explains about the RFID(Radio Frequency Identification) group that describes the pre-requisites for the implementation of IoT. According to this group IoT is a embedded system consisting of uniquely identifiable (addressable) interconnected objects and the addresses to each device are assigned using standard communication protocols.

This paper explains that the IoT driven noninvasive monitoring systems are used for continuous monitoring of patient's physiological status. These systems analyze and store the information using gateways and the cloud. This information is then sent to caregivers for further analysis and review using wireless communication like WSN (Wireless Sensor Network). This system provides a improved quality of continuous care and constant attention towards the patient with less cost and reduces the time required to collect and analyze the data for a caregiver.

a) According to the paper the health sensors can be easily deployed. They are very light weight, inexpensive, easy to monitor and maintain.

b) According to the paper IoT along with e-health sensors can be used to monitor an aging family member.

The above 2 case studies explain about the implementation of IoT using Wireless Sensor Networks and the internet, but in this proposed work the IoT ( consisting of e-health sensors and the ARM-7 microcontroller) is combined with the cloud computing technique to produce best solution for health monitoring. The stored data on the cloud can be accessed anywhere, anytime by the caregivers (health care providers) to take decisions for the best treatment.

This proposed work has several advantages compared to the previous works defined:

1) It provides real time data gathering.

2) Eliminates manual data collection and data entry processes that may include data entry errors.

3) Reduces the requirement of beds occupancy in hospitals as it ensures that, the patient who really needs to get admitted and who can manage at his/her home.

4) Monitoring of many number of patients who are dependent on limited number of medical staff.

5) Patient's health monitoring with minimal cost.

\section{SYSTEM ARCHITECTURE}

The 3 wireless health sensors (Electro Cardio Gram (ECG), heartbeat and temperature) must be connected to ARM7 microcontroller. The microcontroller is responsible for gathering data from sensors and transmitting this data through wireless communication media to a cloud storage service (Dropbox).The Medical staff and patients can make use of this application from different mobile and stationary devices connected to the Internet.

To provide security to the data stored in cloud the following two techniques are used:

a) Secured Socket layer: It establishes a encrypted secured channel between the microcontroller and the web server[2].

b) Using the AES (Advanced Encryption Standard) algorithm, the data will be stored in the encrypted format in the cloud[2].

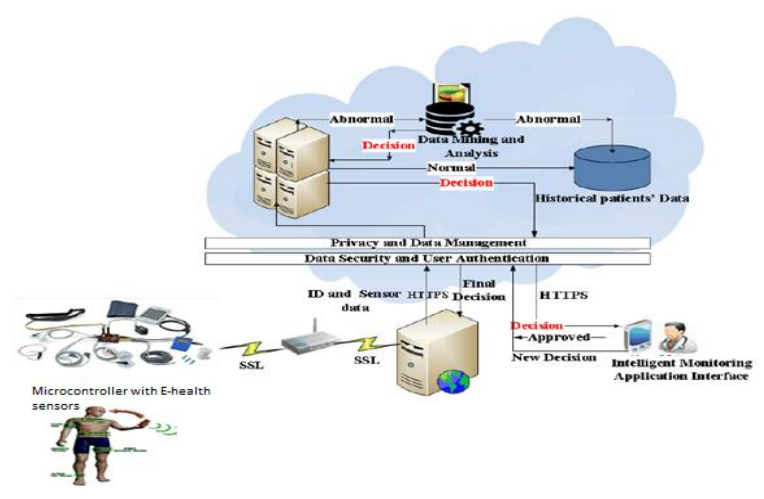

Figure 1: System Architecture

\section{IMPLEMENTATION}

The following hardware components are required to implement the proposed work. The ARM7 microcontroller is a main component that controls and interfaces other components. The GPRS module receives the output from the microcontroller and sends it to the cloud.

1) Microcontroller : ARM7

2) E-health SENSORS: temperature sensor, ECG, Heart beat sensor

3) Power Supply

4) Liquid Crystal Display(LCD)

5) GPRS module

\section{A. ARM7 Microcontroller}

It is a 32bit 64 pin RISC (Reduced Instruction Set Computer) type Microcontroller. Since ARM7 is designed with a very small instruction set and a very small gate count, it is ideal for developing embedded systems. It has low power 
consumption, high performance and takes a tiny amount of the available silicon die area[3].

There are 3 different sensors namely temperature sensor, heart beat sensor, ECG sensor used in this proposed work need to be interfaced with ARM7 microcontroller. The LPC 2129 technology based on this 32 bit ARM7 microcontroller is used for the interfacing[3]. The Microcontroller is built with:

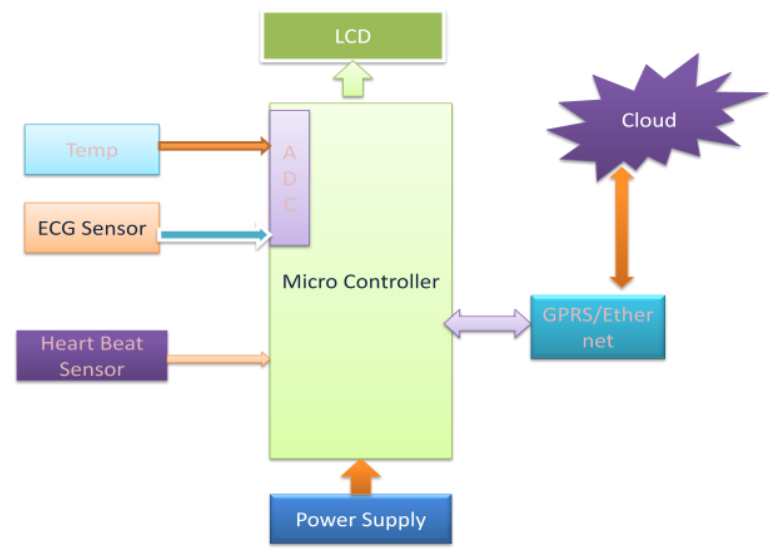

Figure 2: Block Diagram of Proposed Work

1) 128 bit memory interface.

2) $128 / 256 \mathrm{~KB}$ of embedded high speed flash memory.

3) Internal 4-Channel 10 bit ADC (Analog to Digital Converter).

4) 46 GPIO (General Purpose Input Output) lines.

Since most of the sensors are analog sensors (i.e. they produce analog output and this data should be read by the Microcontroller) Microcontroller needs to convert this analog data into digital data. The ARM7 has a built in ADC that converts analog output from the sensors into digital data[3].

B. E-Health Sensors

a) Temperature Sensor

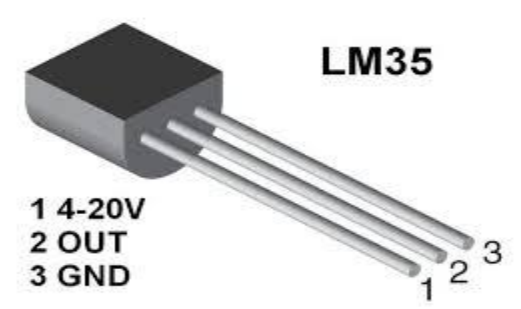

Figure 3: LM35 Temperature Sensor

The LM35(Linear Monolithic series 35) temperature sensor measures temperature more accurately than a thermistor. The voltage produced will be relative to the Celsius temperature. It also possesses a low self heating capability.

\section{b) ECG sensor}

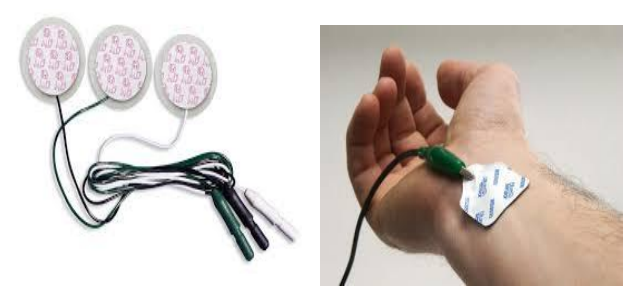

Figure 4: Electro Cardio Gram Sensor

The electrocardiogram (ECG or EKG) is a diagnostic tool. It measures electrical and muscular functions of the heart. The electrocardiogram (ECG) test is commonly used in the diagnosis of a myriad of cardiac pathologies.

c) Heart Beat Sensor

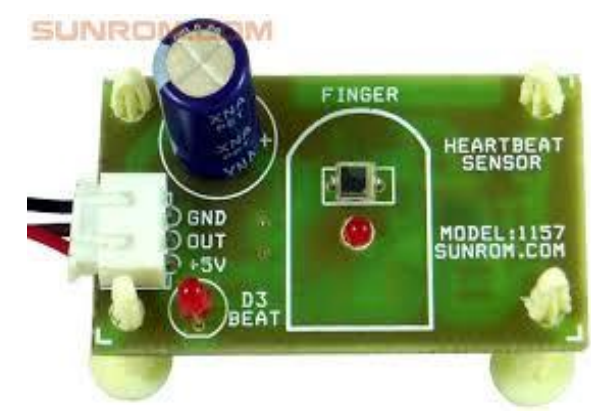

Figure 5:Heart Beat Sensor

When a finger is positioned on the heart beat sensor, it produces a digital output of heart beat. If the sensor is properly working then LED flashes along with the heart beat. This digital output is connected to microcontroller directly to measure the Beats Per Minute (BPM) rate. It works on the principle of light modulation by blood flow through finger at each pulse.

\section{Power supply}

It is a resource of electrical signals. Any device that provides energy in the form of electricity or any other type of energy to a group of loads (or load) is called a power supply unit or PSU. Here in this application we need a $5 \mathrm{~V}$ AC power supply for all electronics involved.

\section{LCD: $16 \times 2$ (Liquid Crystal Display)}

It offers a number of advantages over conventional Cathode Ray Tube(CRT) that makes them ideal for numerous applications. LCD's are flat \& they use only a fraction of power required by CRT. 16X2 LCD is used to display information to users. It displays 16 characters on each of the 2 rows and it has data registers, command registers.

Pin 1 - Ground

Pin $2-+5 \mathrm{~V}$ supply

Pin 3 - not used

Pin 4 - Reset

Pin 5 - Read/write Reads data from microcontroller and writes into LCD

Pin 6 - Enable or disable total LCD operations

Pin 7-14 $\rightarrow 8-$ bit data register. 


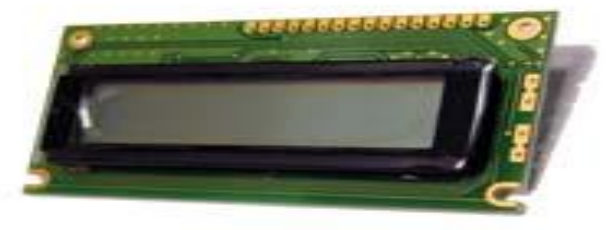

Figure 6: LCD Display

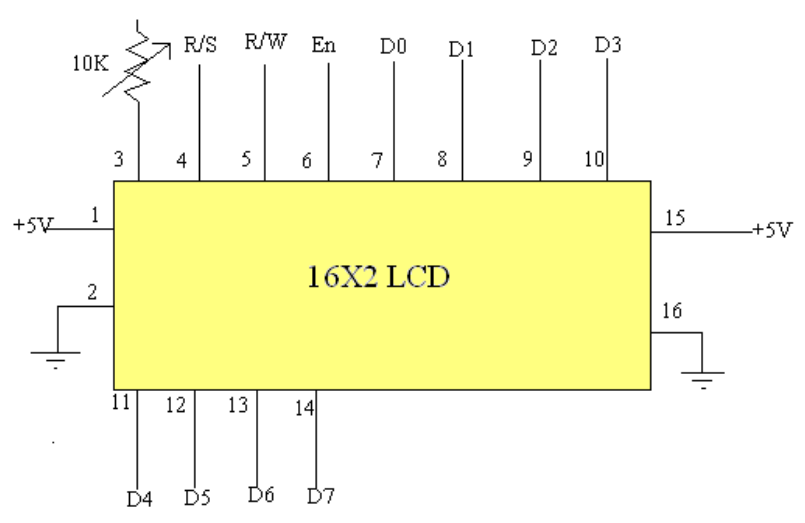

Figure 7: Pin Diagram of LCD

\section{X 2 Alphanumeric LCD Module Features}

1) Intellectual :- It has a built in compatible LCD controller \& RAM that provides plain interfacing

2) $61 X 15.8 \mathrm{~mm}$ viewing area.

3) It can display 224 different symbols

4) Low power consumption ( $1 \mu \mathrm{A}$ typical)

5) Great command set \& user created characters

\section{E. GPRS Module}

Using General Packet Radio Service we can browse internet \& we can get connect to cloud application Drop box. Service providers will charge us based on the data uploaded as well as data downloaded on our mobile phone or computers. The upload and download data charges will be as per standard plans or will be based on per KB data.

\section{Interfacing GPRS with Microcontroller}

The GPRS modules communicate with Microcontroller \& with the computer through UART, to communicate over UART, we just need 3 basic signals which are RXD (receive), TXD (transmit), GND (command ground)

The transmit signal of serial part of Microcontroller is connected with transmit signal (TXD) of the serial interface of GPRS module. The receive signal of Microcontroller's serial port is connected with secure signal (R X D) of serial interface of GPRS module.

The data must be displayed on LCD \& on the connected computer using GPRS module through UART. The
Microcontroller board contains 2 serial interfaces that are UARTO \& UART1. In this proposed work we are using only COM Port 0. The GPRS module is interfaced with the Microcontroller board for communication.(UART0 is a full duplex type of data transmission)

The following application softwares are required to implement this proposed work.

\section{F. Keil Software}

This software tool supports every level of development from the professional application engineers to the student just learning about embedded Software development. The $\mu$ visions 4 Integrated Development Environment (IDE) is a windows based software development platform which allows embedded $\mathrm{C}$ programmes to be written \& executed. The $\mu$ visions 4 debugger to test the applications using C51 compiler \& A51 macro assembler. It offers 2 operating modes [4].

Use simulator mode: - If this mode is set then $\mu$ Vision 4 Debugger acts as a Software only product. It simulates most of the characteristics of the microcontroller without the actual hardware. Using this property of a software we can test \& debug our embedded application before the hardware is ready[4].

Use advanced drivers: - If this mode is set, then the advanced interface will get connect with $\mu$ Vision 3 Debugger directly to emulators called Embedded ICE (on chip Debug System).

\section{G. Flash Magic}

Philips semiconductors produce a variety of Microcontrollers that aspect both on-chip flash memory \& the capacity to be reprogrammed using In-System programming Technology. Flash Magic is windows software from the Embedded systems Academy that allows easy access to all the ISP features provided by the devices.

These features include:

1) Erasing the flash memory (Individual blocks or whole device)

2) Programming the flash memory

3) Modifying the Boot vector \& status Byte.

4) Reading flash Memory

5) Reading \& writing Security bits

6) Direct load of a new baud rate ( high speed communication)

7) Sending Commands to position the device in Boot loader mode

Under windows only one application is able to access COM port at any one time preventing other applications from using the selected COM port. Flash magic only obtains access to the selected COM port, when ISP (Internet Service Provider) operations are getting performed. This means that other applications that need to use COM port such as debugging tools may be used while Flash magic is loading.

\section{CONCLUSION}

The integration of Internet of Things and the cloud computing will create a new generation of technology. This 
combination introduces a better health monitoring system with minimal cost compared to other health monitoring systems. It also improves medical staff performance by helping them to take decisions immediately whenever there is an emergency.

\section{REFERENCES}

[1] R. Buyya, J. Broberg and A. M. Goscinski "Cloud computing: Principles and paradigms", vol.87

[2] Q. Wang, K. Ren, W. Lou, and Y. Zhang, "Dependable and secure sensor data storage with dynamic integrity assurance," in Proc. of IEEE INFOCOM'09, Rio de Janeiro, Brazil, April 2009.

[3] www.atmel.com/Images/DDI0029G_7TDMI_R3_trm.pdf

[4] http://www.keil.com/support/man/docs/uv4/uv4_armcompilers.htm 